\title{
Peertechz
}
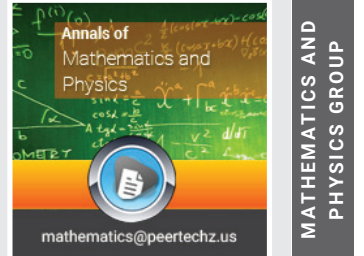

Review Article

\section{An introduction to the superunified theory of quantum fields \& fundamental interactions (Discoveries in pure mathematics)}

Received: 15 October, 2021

Accepted: 18 November, 2021

Published: 19 November, 2021

*Corresponding authors: Besud Chu Erdeni, Unified Theory Lab, Bayangol disrict, Ulan-Bator, Mongolia, E-mail: superunified@yahoo.com

Copyright: @ 2021 Erdeni BC. This is an open-access article distributed under the terms of the Creative Commons Attribution License, which permits unrestricted use, distribution, and reproduction in any medium, provided the original author and source are credited.

https://www.peertechzpublications.com

Unified Theory Lab, Bayangol disrict, Ulan-Bator, Mongolia

Check for updates

\begin{abstract}
This is intended to describe the physical Universe as self-excited and self-organized mathematical continuum. There does exist the universal pure (not applied) mathematical machine perceived by the intelligent observers in a capacity of certain material world. In this short article we are able to indicate only some key points of the theory which suggests practically infinite amount of combinatorics.
\end{abstract}

\section{What is Logic?}

Let us reformulate Newton's First Rule of Reasoning in an extreme miminax form as follows:

\section{Logic is what gets the greatest possible by the least pos-} sible

Then, the least is the mathematical point, that is, Nothing, while the greatest is the Universe which is Something. Consequently, a single point has to be both necessary and sufficient for reconstructing the Cosmos on paper.

Remind that, as Paul Dirac pointed out, the modern physics still remains hopelessly illogical. This is because of the lack of rigorous foundations for both quantum mechanics and general relativity. To correct this situation, we follow Newton's tradition to speculate a theory beginning with any strictest possible definition of what is logic in Nature. In Nature rules the least action principle demanding that, for example, a single postulate should suffice to construct the superunified scheme, if any at all. In addition, the universe must have been evolved instantaneously within the zero time duration. The above minimax principle of logic should act absolutely.

There is Something we call the physical Universe. If logically, at first was Nothing, that is, mathematical point defined by the Euclidean geometry first and later by the Cantor's set theory.
As shown by the entire scientific practice of the $20^{\text {th }}$ century, the existing methods appeared to be either insufficient, or essentially wrong to effectively approach the unification problem. Therefore, "Some fundamentally new insights are certainly needed." (Roger Penrose) [1-6].

Yet, the problem is not so hard for solution as it seems to be. Mathematics has a brilliant new resource. Notably, there does exist the fundamental Theorem of the Universal System of Mathematical Harmony which reads

$$
\boldsymbol{X}^{\Phi \pi e} \boldsymbol{X}=10^{90}
$$

Given that

$$
\begin{aligned}
& \Phi=\frac{\sqrt{2}+1}{2}=1.618033988749 \ldots \\
& \pi=3.141592653589 \ldots ; \\
& e=2.7182818284590 \ldots,
\end{aligned}
$$

we derive a new and, possibly, most fundamental mathematical constant such that

$$
\boldsymbol{X}=\Phi \pi e+1 \sqrt{\mathbf{1 0}^{\mathbf{9 0}}}=1185403.539676801 \ldots
$$


What is crucial, we have revealed the fundamental bifurcation of Mathematical Description. It is required for discerning by the readers the meaning of the following scheme (Scheme 1):

$$
\begin{aligned}
& x^{\Phi \pi e} x=10^{90} \\
& \swarrow \quad \searrow \\
& \text { Analysis Synthesis } \\
& \begin{array}{l}
\text { (Symbolic) } \\
x^{N}-1=0 \quad \Leftrightarrow \quad \boldsymbol{x}^{2}-\boldsymbol{x}-\mathbf{1}=\mathbf{0}
\end{array} \\
& \Downarrow \\
& \text { Radix } 1 \quad \rightleftarrows \quad \text { Metric } \Phi \\
& \Downarrow \\
& e^{(2) \pi i}= \pm 1 \quad \rightleftarrows \quad e^{5 \Phi \pi e i_{1} \sqrt{2}} \\
& \Downarrow \quad \Downarrow \\
& \begin{array}{ccc}
\Psi(x, t) & \Leftrightarrow \quad\left\{\Phi \cdot i_{1}\right\} \\
\searrow & & \swarrow
\end{array} \\
& \text { - Superunified Field Theory - }
\end{aligned}
$$

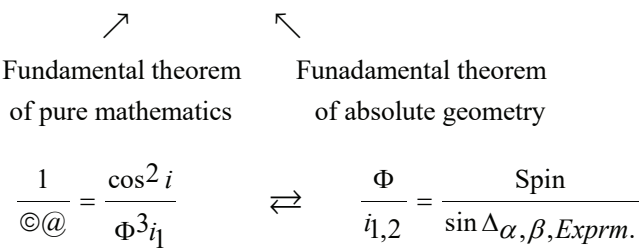

Under the notion of synthesis is meant the final unification of theoretical physics and pure mathematics. This adventure becomes feasible due to the essential completion of Gaussian pure mathematics with new discoveries. In this respect we need to highlight certain consequences of Goedel's Theorem. What is expected to be the final theory of physics, if any, will necessarily be the new and ultimate general theory for physics. But, according to Kurt Goedel's metamathematical theorem on incompleteness of formal systems, the general theory of physics cannot belong to physics itself. It should belong to a higher system that can be pure mathematics of classical type.

The matter is that Newton-Leibniz applied mathematics is still a human invention, while Gaussian pure mathematics unified by the Euclidean system is a natural phenomenon existing a priori. Therefore, there will be no wonder if the physical Universe is described by a numeric theory where everything becomes calculable by elementary algebraic methods, as A . Einstein predicted a century ago.

It is crucial to understand the Logic of Spontaneity and Bifurcation in Nature as being a pure-mathematical phenomenon. The natural logic is spontaneity. Cosmological evolution consists of phase transitions of the initial condition given for cosmogony. The unique way of spontaneous phase transformations is sequential bifurcations, that is, multifurcation of the initial mathematical point.
The initial condition in fundamental theories cannot but be the mathematical point with the imperative consequence that we have to study spontaneous bifurcations of that point and in case we succeed to do this job cosmogony and cosmology will have their rigorous mathematical foundations, not antique mythology coupled with Bible's interpretation.

We now approach the problem of the construction of Absolute Geometry of space-time and matter. For this purpose it will suffice to consider the way of how the Euclidean geometry bifurcates (Figure 1):

What makes difference is how to denote the radius of the circle: Either by the unity 1, or by the golden section constant $\Phi$ keeping in mind the above given fundamental theorem of harmony. In the lower half of the picture we perform an algorithm. The initial condition is a point. It translates and draws a line segment which we denote as $\mathrm{Fi}$, the only nontrivial number which serves a harmonizing factor in the above fundamental theorem. So, the first bifurcation of the given point is complete. The second bifurcation will be rotation of the line segment $\Phi$ around the origin with all the results seen on the previous picture.

Now that we are prompted to come up with certain postulates, including the following two most fundamental

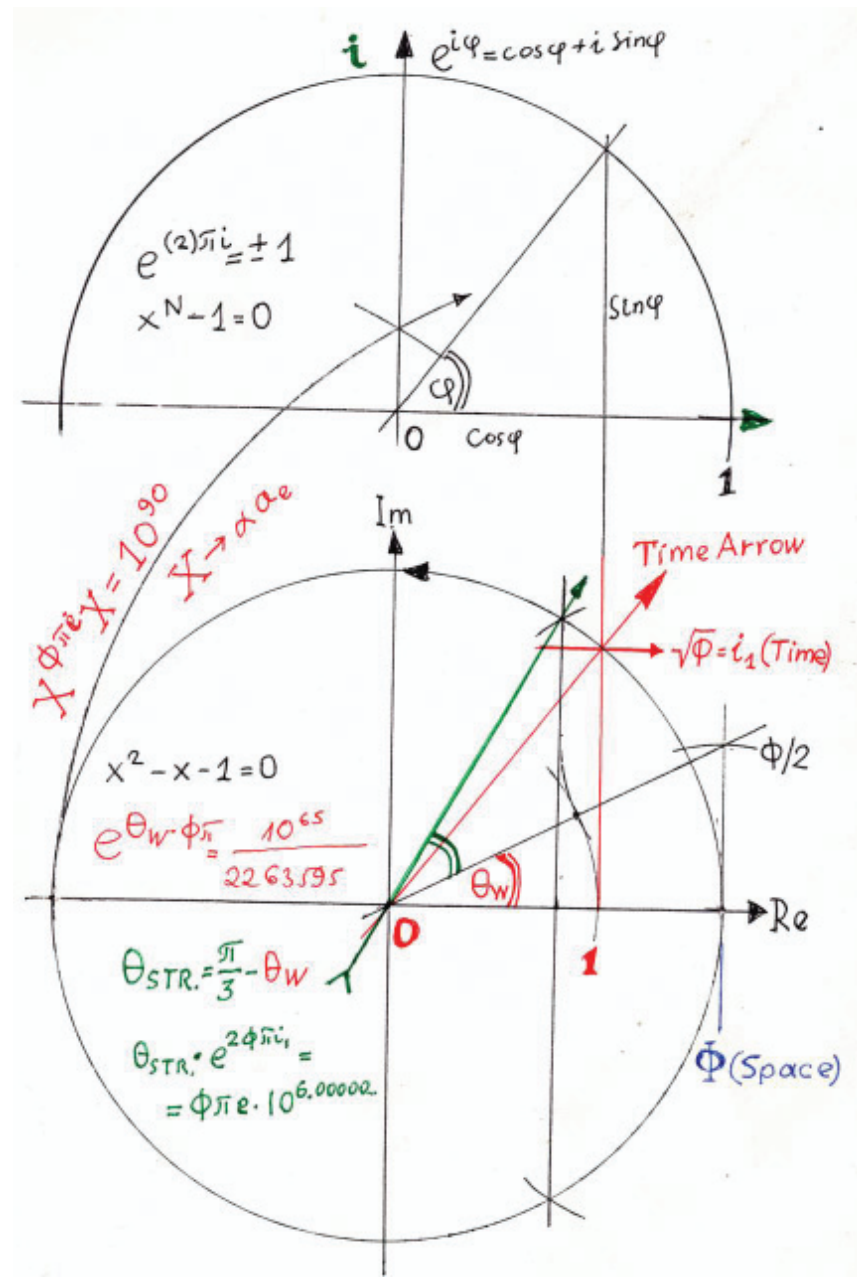

Figure 1: Radius of the circle.

Citation: Erdeni BC (2021) An introduction to the superunified theory of quantum fields \& fundamental interactions (Discoveries in pure mathematics). Ann Math Phys 4(1): 102-111. DOI: https://dx.doi.org/10.17352/amp.000029 
á. $\Phi \equiv$ Newton's absolute mathematical space;

a. $\left\{\sqrt{\Phi}=i_{1}\right\} \equiv$ Newton's absolute, true mathematical time.

Time is an algorithmic bifurcation of space both in geometry and algebra. But this leads to a bit crazy statement that the 4-dim space-time shall simply be

$$
\Phi^{3} i_{1}=5.3883617040570 \ldots
$$

But, as we have seen before, the mathematical method of description of reality is bifurcated, therefore, validating both the analytical and numerical representations of space-time (postulate $\gamma$ )

$$
(x, y, z, t) \Leftrightarrow \Phi^{3} i_{1}
$$

From the philosophical point of view it is a joke that before the Creation act there were neither Greek, nor Latin alphabets. At first were numbers alone.

We should add up yet another two postulates to continue the Standard model as smoothly as possible

$$
\begin{aligned}
& \delta . \Theta_{\text {Weinberg }(\text { electroweak force })}=\arctan 1 / 2 ; \\
& \varepsilon . \Theta_{\text {Nuclear strong force }}=\frac{\pi}{3}-\Theta_{W} .
\end{aligned}
$$

Now we have to try to prove the validity of the $\beta \gamma \delta \varepsilon \alpha-$ Postulates. In any event these postulates are not imagined, but given by compass-and-straightedge algorithms.

The existing quantum-relativistic theories a little bit imprecise compared with the experimental data. This situation reveals itself in the anomaly of the electron magnetic moment which does notably define what we know in physics and what do not know yet. Reference sources provide the experimental value of the magnetic moment anomaly as being close to

$$
a_{\text {Electron }}=0.0011596521
$$

Right at the moment we have learned what is exactly spacetime in Newton's or any other terms. Therefore, immediately,

$$
\frac{1}{a_{\text {Electron }}}=\Phi^{3} i_{1} \sqrt{\mathbf{6 5 8 3 6 1} \cdot 10^{10}}
$$

Whence pure-theoretically

$$
a_{e}=0.0011596520997696346686715329576713
$$

The post-Dirac effect can and must be treated in many other ways available in absolute geometry, for example, (11-13)

$$
\sin \Theta \sqrt{\frac{1}{a_{e}=0.001159652099 \ldots}}=\mathbf{3 6 6 7 4 3 8}
$$

$$
\begin{gathered}
\sin \Theta_{W} \cos \Theta_{W} \sqrt{\frac{1}{a_{e}=0.0011596521000 \ldots}}=\mathbf{2 1 8 3 6 3 8 3} \\
\cos 2 \Theta_{S T R} \sqrt{\frac{1}{a_{e}=0.0011596521000 \ldots \cdot \cos 2 \Theta_{S T R .}}}=\mathbf{3 2 0 9 0 6 4 5 7}
\end{gathered}
$$

Each of these calculations takes about 10 seconds compared with 10 years of work on supercomputers by the group of Toichiro Kinoshita lately. And it is not joke, but paradigms change and change abruptly as ever in the history of sciences.

Does the above samples mean that Leopold Kronecker is going to be true with his mythological sentence: "Die ganzen zahlen hat der liebe Gott gemacht, alles andere est Menschenwerk." ?

All this is going to be true if only the bold integers occurring in the above are not by a blind chance. The integer 658361 that defines the subtle effect, $a_{e}$, is any doubt fundamental. Consequently, we have to search for some more fundamental reasons of the absolute system of mathematical harmony behind it. Indeed,

$$
\mathbf{6 5 8 3 6 1} \cdot e^{5 \Phi \pi e i_{1} \sqrt{2}}=\frac{\mathbf{1 8 6 5 9 2 1}}{3} \cdot 10^{54} .
$$

The character of the system is seen in

$$
2 \sin \lg \left\{\mathbf{6 5 8 3 6 1} \cdot e^{5 \Phi \pi e i_{1} \sqrt{2}} \cdot \frac{3}{\mathbf{1 8 6 5 9 2 1}}\right\}=\Phi .
$$

where true decimal digits are

$$
\Phi=1.61803398874 \ldots
$$

The generel problem in physics is now how to continue the Standard Model toward superunified field theories. For this purpose the following operator immediately avails itself:

$$
e^{\Phi \pi \cdot \Theta_{W}}=\frac{10^{65}}{\mathbf{2 2 6 3 5 9 5}} .
$$

The electroweak Theta has whatever possible connections to the Strong force Theta, including

$2263595 \cdot 2 \Theta_{S T R .}=151366366$

The integer on the right side is not accidental, but owes to

$$
\mathbf{1 5 1 3 6 6 3 6 6} \cdot e^{5 \Phi \pi e i_{1} \sqrt{2}}=\frac{\mathbf{1 2 8 7 0 0 3 7}}{9} \cdot 10^{56}
$$

where true decimal places are

$$
\pi=3.1415926535 \ldots
$$

Apply to the fundamental theorem and compute

$$
12870037 \cdot \Phi \pi e=\frac{1600494919}{9}
$$

Citation: Erdeni BC (2021) An introduction to the superunified theory of quantum fields \& fundamental interactions (Discoveries in pure mathematics). Ann Math Phys 4(1): 102-111. DOI: https://dx.doi.org/10.17352/amp.000029 
The whole numbers we encounter we name Harmonious Integers (HI). HIs do notably serve the main tool of selfconsistence and provability of the entire system of universal mathematical harmony. Without them we were unable to compute the anomaly of electron magnetic moment. So long as eliminating the post-Dirac paradox, we havevalready validated the unified field theory and we may stop on this with quiet conscience. Yet, the superunification theory suggests practically infinite amount of combinatorics simply by the reason that it should be theory of everything, including, say, the velocity of light.

It is a bit early to abolish Newton's absolutism in physics. Special relativity theorists could not imagine to write an obvious

$$
\frac{\text { Absolut space }}{\text { Absolute time }}=\text { absolute velocity }
$$

Absolute time

In terms of absolute geometry

$$
\begin{aligned}
& \frac{\Phi}{i_{1}}=i_{1} \\
& \operatorname{dim}_{\Phi} c=i_{1}
\end{aligned}
$$

Measurement units in physics are intercorrelated. So, imagine that true experimental numbers like 299792458 should in depth be theoretical numbers in a way or other. Indeed, for example,

$2997924588^{\pi}=\mathbf{4 2 7} 285 \cdot 10^{20.99999999910 \ldots}$

If accurately,

$$
\sqrt[\pi]{\mathbf{4 2 7 2 8 5 \cdot 1 0 ^ { 2 1 }}}=\mathbf{2 9 9 7 9 2 4 5 8 . 1 9 7 3 3 1 0 2 6 1 2 0 \ldots}
$$

Violation of mathematical symmetries here and elsewhere owe to the spontaneous self-perturbation effects of the entire system. Thus, higher order approximations are always possible and elementary within the system.

In the dimensional case

$$
c i_{1}=100 \exp \exp e\left\{1-\frac{1}{4434.531539}\right\}
$$

The formula is corrected among others because of

$$
\left\{\frac{\mathbf{5 2 5 6 7 0 9 3 8 5}}{4434.531541}\right\}^{\Phi \pi e+1}=\mathbf{1 0}^{\mathbf{9 0}}
$$

It should be pointed out that in fact there are two anomalies in physics:

$\alpha_{\text {Sommerfel }}=137.035999$

$a_{\text {Electron }}=0.0011596521$.

and we postulate that the space and time vectors are disarrayed either by the straight angle (electromagnetic waves) or by the angle inverse to the fine structure constant yielding spinor particle structure

$$
\Delta_{\text {Exprm. }}=\frac{1}{\alpha_{\text {Sommerfeld }}}=137.035999 ;\left\{\Phi \Delta_{\text {Exprm. }} .{ }_{1}\right\}^{\Phi \pi e}=\frac{\mathbf{6 4 8 1 6}}{9} \cdot 10^{30}
$$

At first the space-time separation angle Delta was

$$
\frac{360}{\Phi^{2}}=\Delta_{1}
$$

before it undergoes perturbative transformations like

$$
\cos 2 \Theta_{S T R} \sqrt{\frac{\left\{\frac{\Delta_{1}}{\Delta_{\text {Exprm. }}=137.036000 \ldots}-1\right.}{\cos 2 \Theta_{\text {STR. }} .}}=\frac{\mathbf{4 0 2 1 6 9 5 9}}{2} .
$$

Due to the universality of the system there are many other ways of approximating to the experimental Delta.

What overrides is the general theorem of the universal system of mathematical harmony

$$
X=\Phi \pi e+1 \sqrt{\mathbf{1 0}^{\mathbf{9 0}}}=1185403.539676801580 \ldots
$$

In this regard it should be pointed out that infinite fractions like $X$ have no meaning exempt formal exactitude. By this reason the universal mathematical machinery works as simply as any 10-digit electronic calculator in terms of the first order approximations. So, we will shorten and memorize the $X$ as

$$
X=1185403.54 .
$$

What is crucial to be discerned, the mathematical symmetries defined by this new constant are broken in the physical world which case is manifested by the existence of the two anomalies in the form of the Sommerfeld and Dirac constants. Therefore, we are asked to write and an evident

$$
\begin{gathered}
\frac{X_{1185403.54} \cdot \alpha_{1 / 137.035999^{a} e(0.0011596521)}}{10}= \\
=1+\frac{1}{319.0063275} .
\end{gathered}
$$

and compute the self-perturbation effect of the entire system as

$$
\frac{\mathbf{3 7 8 1 5 1 2 3 0}}{\boldsymbol{X}=1185403.54}=319.0063277 ; \quad \frac{\mathbf{3 2 2 9 3 1 1 0 0 0}}{\mathbf{3 7 8 1 5 1 2 3 0}}=\pi e
$$

Since the quantum-relativistic theories of physics are true on their own, both these anomalies look rather a problem of mathematics than physical. If so, anomalies may come from the incompleteness of the existing mathematics. We have completed pure mathematics at least by the constant, $X$. As a result we enter the new virgin land of the universal system of harmony. Now we are enabled to guess that the anomalies in physics under discussion arise because of some harmonious reasons. They are like lubrication substances in the mechanism

Citation: Erdeni BC (2021) An introduction to the superunified theory of quantum fields \& fundamental interactions (Discoveries in pure mathematics). Ann Math Phys 4(1): 102-111. DOI: https://dx.doi.org/10.17352/amp.000029 
of the universal machinery. Therefore, the problem can be clarified as above and elsewhere. The $\Phi \pi e$-engine of the universal system cannot but be self-exited and thus bifurcated into some phenomena like the two anomalies that make physicists' life so miserable.

Experimenting with the system, one will reveal how many equations end up with the following result:

$$
\frac{1}{10} \ln \lg \left\{\left\{\exp \frac{1}{\left\{\Delta_{\text {Exprm. }}-\mathbf{1 3 7}\right\}}\right\} \cdot \frac{1}{a_{\text {Electron }}}\right\}+1=\frac{\Phi \pi}{4}
$$

It describes the fermion-boson process in general using notations

$$
\begin{aligned}
& \text { Fermion }=\frac{\Phi \pi}{4} \\
& \text { Boson }=10\left\{\frac{\Phi \pi}{4}-1\right\} .
\end{aligned}
$$

It is derivable as consistently as

$$
F B=\ln \lg \left\{\pi \Phi^{3} \sqrt{i_{1} i_{2}} \cdot 10^{29.9999 \ldots}\right\}
$$

A pecularity of absolute geometry is that Newton's mathematical time is bifurcated, too, giving the global cosmological absolute time

$$
\sqrt{\Phi}=i_{1}
$$

and local relative time

$$
i_{2}=\frac{4}{\pi}
$$

Consequently, the mean cosmological time shall certainly be

$$
\sqrt{i_{1} i_{2}}
$$

Therefore, we are required to write the four-dimensional curved and torsional absolute/relative Newton/Einstein spacetime of absolute geometry in the form

$$
\pi \frac{\Phi^{3} \sqrt{i_{1} i_{2}}}{\operatorname{Spin}_{\cos 30}}
$$

It should be reminded that in classical quantum mechanics

Spin $=\cos 30^{\circ}$

As for the dimensionality problem, we will obviously have

$$
\begin{aligned}
& \operatorname{dim}_{\Phi} \Phi^{3} i_{1}=3 \frac{1}{2} \\
& \text { Cartesian }=(3+1) .
\end{aligned}
$$

So, an essentially complete representation of the cosmic space-time has to be written as

$$
\pi \frac{\Phi^{3} \sqrt{i_{1} i_{2}}}{\operatorname{Spin}_{\cos 30}}\left\{(3+1) \operatorname{dim}_{\Phi} \Phi^{3} i_{1}\right\}=273.7865863
$$

It asks to be enhanced to whatever extent, including

$$
\left\{\pi \frac{\Phi^{3} \sqrt{i_{1} i_{2}}}{\operatorname{Spin}_{\cos 30}}\left\{(3+1) \operatorname{dim}_{\Phi} \Phi^{3} i_{1}\right\} \frac{\Delta_{\text {Exprm. }}}{\sin \Delta_{\text {Exprm. }}}\right\}
$$

The existing pure mathematics ends up with the Euler's rotation operator which bifurcates into whatever super and ultra operators of absolute geometry $\left\{e^{(2) \pi i}= \pm 1\right\} \Rightarrow e^{\Phi \pi i_{1}} \rightarrow e^{2 \Phi \pi i_{1}} \rightarrow \ldots \rightarrow e^{5 \Phi \pi e i_{1} \sqrt{2}} \rightarrow \ldots$

Therefore, one of standard ways of derivation of things is

$$
\begin{gathered}
\left\{\pi \frac{\Phi^{3} \sqrt{i_{1} i_{2}}}{\operatorname{Spin}_{\cos 30}}\left\{(3+1) \operatorname{dim}_{\Phi} \Phi^{3} i_{1}\right\} \frac{\Delta_{\text {Exprm. }}}{\sin \Delta_{\text {Exprm. }}}\right\} . \\
\left\{\boldsymbol{X} \cdot e^{5 \Phi \pi e i_{1} \sqrt{2}} \cdot \frac{\exists}{\odot @}\right\}=\frac{10^{69.0000 \ldots}}{e^{\Phi \pi i_{1}} .}
\end{gathered}
$$

(The third component in the second brackets will be explained later.)

Previously we have demonstrated the workability and efficiency of the formal system of absolute geometry discovered, however, too long after Euclid and Gauss.

It is time to formulate the general rule of absolute geometry. Any true formulae and equations written on the left side of the equality sign will give whole numbers or fundamental parameters on the right. Those whole numbers in turn have to be justified as not accidental by means of the absolute numeric calculus, being the system of universal mathematical harmony. For example,

$$
\begin{gathered}
\cos \Theta_{W} \sqrt{299792458 i_{1}}=\cos 2 \Theta_{S T R .} \cdot 10^{10.00000 \ldots} \\
\cos 2 \Theta_{S T R} \cdot \sqrt{299792458} i_{1}=\frac{10^{26.0000000 \ldots}}{\mathbf{1 4 2 7 6}}
\end{gathered}
$$

It is also foreseeable that

$$
\otimes^{2} \Delta_{1} \equiv c
$$

It is because of the existence of a logarithmic operator such that

$$
10^{\otimes}=10000 \otimes
$$

By analogy

$$
e^{\oplus}=1000 \oplus
$$

Interested students are required to calculate the values of the two operators by iteration and approximate 


$$
\pi \frac{\Phi^{3} \sqrt{i_{1} i_{2}}}{\text { Spin }} \equiv \sqrt[\otimes]{10 e^{5 \Phi \pi e i_{1} \sqrt{2}}}
$$

The meaning of the previous equation is that it derives the four-dimensional space-time from the ultra-operator of the major mathematical symmetries given by

$$
e^{5 \Phi \pi e_{1} \sqrt{2}}
$$

What acts is logarithmic operators

$$
\begin{aligned}
& \otimes=4.66924683287774767030699969 \ldots ; \\
& \oplus=9.118006470402740121258337182 \ldots
\end{aligned}
$$

The system we consider is universal. Dictionary definition of universality reads: Universality is the quality of being true in or appropriate for all situations. It is by this reason that combinatorics of the unification theory is practically infinite. Universality of the absolute calculus makes us to feel the embarrassment of the riche which is, however, not very pleasant feeling. We risk to be lost in the infinite wealth of the resource offered by the absolute geometry. Therefore, we are required to be wise and witty enough to distinguish from the beginning what is the major selected theorems of the superunified theory and what is just a game. The ultimate goal of physics is to derive space-time, mathematically and from fundamental principles. Therefore,

$$
\pi \frac{\Phi^{3} \sqrt{i_{1} i_{2}}}{\pi \cdot \sqrt[\oplus]{10 e^{5 \Phi \pi e i_{1} \sqrt{2} .00000 \ldots}}}
$$

Newton's time is bifurcated into absolute global cosmic and relative local times by some subtle algorithmic reasons we here omit. At least we have an exact

$$
\sin \Delta_{\text {Exprm. }} \sqrt{\left\{i_{2}=\frac{4}{\pi}\right\}^{\Delta_{\text {Exprm. }}}}=\mathbf{1 2 4 2 0 1} \cdot 10^{16}
$$

Physics is puzzled for a century by the quantum leap processes in the micro world. So, let us consider the topic of quantum leap operators \& cosmological evolution.

The best way to scare away the useless people and get rid of homegrown philosophers is to say that even elementary algebra still remains incomplete and we are going to complete it.

Once I wanted to have some specified number like

$$
\text { ABCdef } \ldots=\frac{1}{C B A d e f \ldots}
$$

and got it as

$$
\begin{aligned}
& \exists=\mathbf{5 7 1 1 0 0 5 2 2 ~ 6 4 7 \ldots ;} ; \\
& \frac{1}{\exists}=\frac{1}{\mathbf{1 7 5 1 0 0 5 2 2 6 4 7 \ldots}}
\end{aligned}
$$

and named it, though tentatively, as the bifurcation constant.
Geometry needs also in quantum leap operators such as

$$
\begin{aligned}
& e^{\bigodot}=\bigodot^{-1} ; \\
& \ln (=-\odot ; \\
& \bigodot \odot=0.567149290405 \ldots
\end{aligned}
$$

One can calculate yet another obvious one by iterations

$$
\begin{aligned}
& \lg @=-@ ; \\
& 10^{@}=@^{-1} .
\end{aligned}
$$

The most usable decimal form of the mirror-E is

$$
\exists=5.71100522647 \ldots
$$

Now that we are prompted to write the following obvious tri-unity of the above operators

$$
\frac{\exists}{(\odot) ~}
$$

This will be the integrated operator of cosmological evolution.

Their does exist also an operator of organic growth process

$$
\begin{aligned}
& \chi=5.06384686161429420 \ldots \\
& \exp e^{\chi}=\chi \cdot 10^{68}
\end{aligned}
$$

Generally, organic growth and Phi-invariance are equivalent concepts, for

$$
e^{e e} \equiv 1000 \Phi
$$

As it is nearly a common knowledge, the purpose of the Universe is self-cognition. In this regard we have before obtained the three fundamental operators of mathematical harmony and symmetries, and that of spontaneous bifurcation evolution of the entire system

$$
\begin{array}{r}
\text { Universal harmony } \\
\text { Fundamental symmetries } e_{e}^{\Phi \Phi e+1} \sqrt[5 \pi i_{1} \sqrt{2}]{ } ; \\
\begin{aligned}
\text { Quantum mechanism of evolution } \\
\frac{\exists}{\odot @} .
\end{aligned}
\end{array}
$$

They compose the Holy Trinity of geometry

$$
\left\{\boldsymbol{X} \cdot e^{5 \Phi \pi e i_{1} \sqrt{2}} \cdot \frac{\exists}{(\odot @)}\right\}=
$$

$=2.8262258124126953859726921782076 \mathrm{e}+61$

It is notably this trinity that rules the world. And the world looks to us hardware, though in fact it is pure software we are trying to describe. The trick of making the observers cheated is the method of topological configurations. The five Fermat- 
Gauss regular polygons provide a configuration which is topological density

$$
\{3,5,17,257,65537\}_{+}^{\times} \text {. }
$$

It is a decent mathematical fact owing to

$$
\sqrt[\pi]{\{3,5,17,257,65537\}_{+}^{\times 5}}=10^{22.9999 \ldots}
$$

And an extremely favourable theorem of the unification theory appears to be, by definition,

$$
\left\{\boldsymbol{X} \cdot e^{5 \Phi \pi e i_{1} \sqrt{2}} \cdot \frac{\exists}{(@ @)}\right\} \equiv\{\mathbf{3}, \mathbf{5}, \mathbf{1 7}, \mathbf{2 5 7}, \mathbf{6 5 5 3 7}\}_{+}^{\times} .
$$

It implies that whatever does the holy trinity in geometry reduces in the end, however, to some compass-andstraightedge algorithmic constructions in plane projections like in Figure 1. Indeed, the unified model of fundamental fermion particles can and must be drawn on a complex plane as multifurcation of the mathematical point. If accurately,

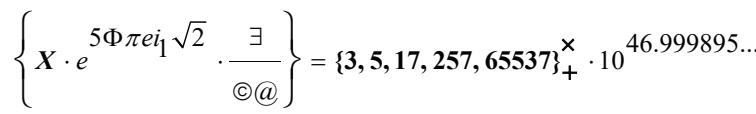

The equation is corrected by

$$
\overline{4165.1048983637799713367811370364}
$$

As it is easy to guess,

$$
e^{\Phi \pi i_{1}} \cdot 4165.1049=\frac{\mathbf{2 4 0 9 8 4 0 7}}{9}
$$

The Platonic world of regular figures owes to

$$
\mathbf{D}\{\mathbf{2}\}_{+}^{\times}=\{\mathbf{3}, \mathbf{5}, \mathbf{1 7}, \mathbf{2 5 7}, \mathbf{6 5 5 3 7}\}_{+}^{\times}=282690452389605 ;
$$

$$
D\{3\}_{+}^{\times}=\begin{array}{|c|c|c|}
\hline 4 & 6 & 4 \\
\hline 6 & 12 & 8 \\
\hline 8 & 12 & 6 \\
\hline 12 & 30 & 20 \\
\hline 20 & 30 & 12 \\
\hline
\end{array} \quad=313714645401600000
$$

$$
\mathrm{D}\{4\}_{+}^{\times}=\begin{array}{|c|c|c|c|}
\hline 5 & 10 & 10 & 5 \\
\hline 8 & 24 & 32 & 16 \\
\hline 16 & 32 & 24 & 8 \\
\hline 24 & 96 & 96 & 24 \\
\hline 120 & 720 & 1200 & 600 \\
\hline 600 & 1200 & 720 & 120 \\
+
\end{array}=
$$

$$
=2.8338438986259070341838012416 \cdot 10^{45} \text {. }
$$

In terms of absolute geometry the Pythagoras-Plato world has to be written in short as

$$
\Phi^{3} i_{1} \cdot \mathbf{D}\{\mathbf{4}\}_{+}^{\times}
$$

And if in shortest, the quantum Big Bang event is written as simply as

$$
\frac{10^{45.99999 \ldots}}{\odot}=\frac{\Phi^{3} i_{1}}{\operatorname{Spin}} \cdot \mathbf{D}\{4\}_{+}^{\times} \text {. }
$$

Fundamental theories are obliged to derive their constants from basic principles, and explicitly. Relativity theories are deficient to accomplish this task. The unified theory makes difference. E.g., we achieve Newton-Gauss unification in a nontrivial way

$$
\begin{aligned}
& \mathbf{6 5 5 3 7}^{\mathbf{6 . 6 7 3}}=\Phi \pi e \cdot 10^{30.9999 \ldots} ; \\
& \left\{G^{G}\right\}^{G}=\Phi \pi \cdot 10^{35.999999 \ldots .}
\end{aligned}
$$

Newton's bare numeric gravitational constant can be derived in many ways and proved that it infinitely tends to the finite fraction 6.673. Absolute dimension of $\mathrm{G}$ is

$$
\operatorname{dim} G=\Phi^{2} \sqrt{2}
$$

Therefore, we are prepared to configure the selfgravitational four-dimensional curved and torsional Pythagoras-Plato space-time as below:

$$
\left.\arccos \lg \lg \lg \frac{\langle\operatorname{dim} G \cdot}{\left\{\pi \frac{\Phi^{3} \sqrt{i_{1} i_{2}}}{\operatorname{Spin}}(3+1) \operatorname{dim}_{\Phi} \Phi^{3} \sqrt{i_{1} i_{2}}\right\} .}\right\rangle
$$

This strange logarithmic mechanism is somewhat standard in geometry owing to the radix problem of mathematics seen in

$$
\Phi^{10}+\Phi^{-10}=\mathbf{1 2 3}
$$

It is compulsory that we investigate what is the phenomenology of the modern physics from the point of view of unification theory. We use the following experimental values of the five fundamental constants of physics:

$$
\begin{aligned}
& c_{\text {Maxwell }}=2.99792458 ; \\
& G_{\text {Newton }}=6.673 ; \\
& h_{\text {Planck }}=6.62606876 ; \\
& m_{\text {electron }}=9.10938188 ; \\
& e_{\text {Coulomb }}^{ \pm}=1.602176462 .
\end{aligned}
$$

The absolute-geometric dimensions are

Citation: Erdeni BC (2021) An introduction to the superunified theory of quantum fields \& fundamental interactions (Discoveries in pure mathematics). Ann Math Phys 4(1): 102-111. DOI: https://dx.doi.org/10.17352/amp.000029 


$$
\begin{aligned}
& \operatorname{Dim} G=\frac{\Phi^{2}}{\sqrt{2}} ; \\
& \operatorname{Cosm} .=\frac{G}{\operatorname{dim} G} ; \\
& \operatorname{Dim} m=\sqrt{2} ; \\
& \operatorname{Dim} e^{ \pm}=\Phi \sqrt{\sqrt{2}} ; \\
& \operatorname{Dim} c=i_{1} ; \\
& \operatorname{Dim} E=\Phi \sqrt{2} .
\end{aligned}
$$

The phenomenology as a whole has to be configured as obviously as

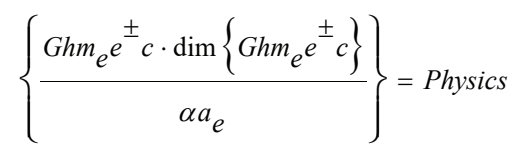

Because of the universality of the system, it can be derived in many equivalent ways. Most standardly

$$
\{\text { Physics }\}^{\Phi \pi i_{1}}=\frac{\mathbf{1 6 5 9 5 7 9 4}}{9} \cdot 10^{56}
$$

The HI on the right is justified by such consistent reasons of the system as

$16595794 \cdot \Phi \pi e=229313715$

$\left\{\pi \frac{\Phi^{3} \sqrt{i_{1} i_{2}}}{\operatorname{Spin}} \cdot(3.5 \times 4)\right\} \cdot \mathbf{1 6 5 9 5 7 9 4}=\mathbf{4 5 4 3 7 0 5 7 8 6}$.

Physicists do mainly think that the final theory should provide no more and no less than the theory of elementary particles. It is in 1987 that I came to the idea that fundamental fermions are nothing but different excitation states of the mathematical point.

Now the theory of elementary particles is fully embraced by the configuration of the internal geometry of the pointsingularity

$$
\left\{\frac{\Delta_{\text {Exprm. }} \Theta_{W} \cdot 2 \Theta_{\text {STR. }}}{\sin \Delta_{\text {Exprm. }} \sin \Theta_{W} \cos \Theta_{W} \cos 2 \Theta_{\text {STR. }}}\right\}
$$

It owes to a standard mechanism

$$
\begin{aligned}
& \left\{\frac{\Delta_{\text {Exprm. }} \Theta_{W} \cdot 2 \Theta_{\text {STR. }}}{\sin \Delta_{\text {Exprm. }} \sin \Theta_{W} \cos \Theta_{W} \cos 2 \Theta_{\text {STR. }}}\right\}^{\Phi \pi e}= \\
& =\sin \Delta_{\text {Exprm. }} \cdot 10^{87.999 \ldots .}
\end{aligned}
$$

Or else,

$$
\begin{aligned}
& \left\{\frac{\Delta_{\text {Exprm. }} \Theta_{W} \cdot{ }^{2 \Theta_{S T R .}}}{\sin \Delta_{\text {Exprm. }} \sin \Theta_{W} \cos \Theta_{W} \cos 2 \Theta_{S T R .}}\right\} . \\
& \left\{X \boldsymbol{X} \cdot e^{5 \Phi \pi e i_{1} \sqrt{2}}\right\}=\frac{10^{65.999999 \ldots}}{\cos 2 \Theta_{S T R},} .
\end{aligned}
$$

The real-imaginary bifurcation in mathematics is

$$
\cos i \Leftrightarrow \cos 2 \Theta
$$

Symmetries are necessarily broken

$$
\left\{10 \cos 2 \Theta_{\text {STR. }}\right\}^{2} \neq 10 \cos i
$$

One of Ramanujan famous series embraces the entire continuum and equals suddenly to

$$
\sqrt{\frac{\pi e}{2}}
$$

We study the continuum, too, which case obliges us to have

$$
\left\{\sqrt{\frac{\pi e}{2}}\right\}^{\Phi}=2 \Phi\left\{1-\frac{\cos 2 \Theta_{S T R .}}{100000}\right\}
$$

Consequently, the strong Theta parameter of geometry is the measure of symmetry violations and broken symmetries to be duly compensated by the existence of the physical Universe. The strong-Theta angle is always doubled, probably, by the reasons known in chromodynamics.

In the superunification theory it is easy to write whatever large superunified equations. Yet, logically and aesthetically more important to derive compact and deepest in meaning equations. Therefore, the next one shows in briefest how spacetime of absolute geometry is factorized into most essential configurations of the system of universal mathematical harmony

$$
\begin{aligned}
& \left\{\boldsymbol{X} \cdot e^{5 \Phi \pi i_{1} \sqrt{2}} \cdot \frac{\exists}{\odot @}\right\} \cdot \\
& \left\{\frac{\Delta_{\text {Exprm. }} \Theta_{W} \cdot 2 \Theta_{S T R .}}{\sin \Delta_{\text {Exprm. }} \sin \Theta_{W} \cos \Theta_{W} \cos 2 \Theta_{S T R .}}\right\} \\
& \left\{\frac{\left.G \cdot h m_{e^{e^{ \pm}} c \cdot \operatorname{dim}\left\{G h m_{e} e^{ \pm} c\right\}}^{\alpha a}\right\}=}{\alpha a_{e}}\right\} \\
& =\pi \frac{\Phi^{3} \sqrt{i_{1} i_{2}}}{\operatorname{Spin}}\left\{(3+1) \operatorname{dim}_{\Phi} \Phi^{3} \sqrt{i_{1} i_{2}}\right\} \cdot 10^{75.000 \ldots .}
\end{aligned}
$$

The space-time structure of electromagnetic waves is

$$
\Phi \perp i_{1}
$$

Then, spinor particles will be 
$\Phi \cdot 137.035999 \cdot i_{1} \cdot$

(109)

Now we prepared to write a full blown unit particle as follows below:

$$
\begin{aligned}
& \left\{{ }^{\text {Space }} \Phi \cdot 90 \cdot 137.035999 \cdot{ }^{\text {Time }} i_{1}\right\} \\
& \cdot G h m e^{e^{ \pm}} c \cdot \operatorname{dim}\left\{\text { Ghm }_{e} e^{ \pm} c\right\}=\sqrt[\pi]{\mathbf{1 4 2 7 3 6} \cdot 10^{23}}
\end{aligned}
$$

And note that

$$
142736 \cdot\{3,5,17,257,65537\}_{+}^{\times} \cdot \frac{7434925}{3}=10^{26} .
$$

The cosmlogical constant of the unified theory is

$$
\text { Cosm. }=\frac{G}{\operatorname{dim} G} .
$$

What is strange, in this theory Einstein's energy is almost absent. Instead, the theory everywhere uses the universal energy configuration

$$
\mathbf{U}_{\mathbf{E}}=E_{a g} \cdot h h_{a g} \cdot 2 \mathrm{~N} m_{e} c^{2} \cdot \frac{2 \Theta_{S T R .}}{\cos 2 \Theta_{S T R .}}=.
$$

\section{$=2260258601.268245380 \ldots$}

And this notably explains some oddities of observations making much noise in cosmology and astrophysics

$$
\left\{\mathbf{U}_{\mathbf{E}}\right\}^{\text {Cosm. }}=10^{e} \cdot 10^{31.00000 \ldots}
$$

Or else,

$$
\left\{\mathbf{U}_{\mathbf{E}}\right\}^{\oplus .}=\pi \frac{\Phi^{3} \sqrt{i_{1} i_{2}}}{\operatorname{Spin}} \cdot 10^{83.99999 \ldots} .
$$

These describe the laws of cosmological expansion.

Discovering absolute geometry, the human civilization passes over the Pons Asinorum in cognition. In this article we indicated only some key points of physics leaving aside many sections of the theory, including the theory of biology in general and human genetics, in particular.

To around up, we would highlight two points related to the cognitive history. The best idea of the Occidental civilization is neither geometry, not monotheism, but the concept of geometrizing God. We have restored the Creation Act algorithm performed once by the Supreme Intelligence. Perhaps, no human cowardice could ever argue with this revolutionary fact, though something has gone deeply wrong in this world lately.

The most comprehensive and prophetic scientific program was and still remains General Scholium of Newton. Indeed, as it proves to be, the "eternal, infinite, absolutely perfect" cosmic intelligence does exist.

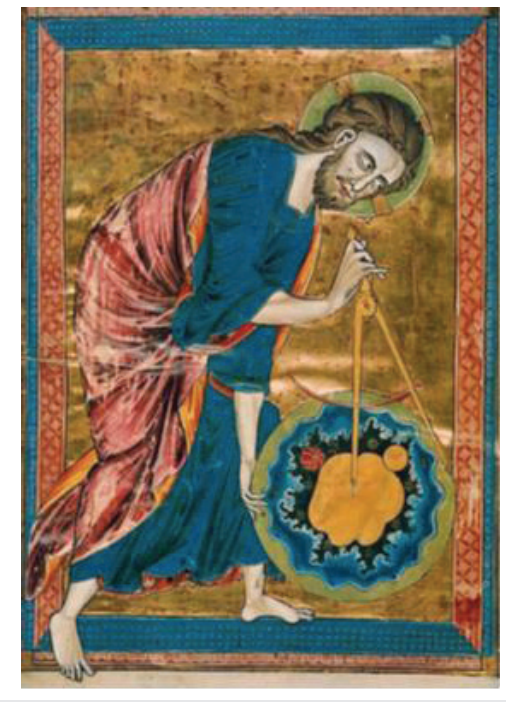

Newton's immortal soul may now eventually relax seeing that the universal gravitation is golden algorithmic origin

$$
\left\{\pi \frac{\Phi^{3} \sqrt{i_{1} i_{2}}}{\operatorname{Spin}}\right\}^{G \cdot \operatorname{dim} G}=\cos \Theta_{W} \cdot 10^{16}
$$

In cosmological terms

$$
\begin{gathered}
\left\{\pi \frac{\Phi^{3} \sqrt{i_{1} i_{2}}}{\operatorname{Spin}} \cdot(3+1) \cdot \operatorname{dim}_{\Phi} \Phi^{3} \sqrt{i_{1} i_{2}}\right\}^{\operatorname{Cosm}=\frac{G}{\operatorname{dim} G}}= \\
=\frac{\mathbf{1 2 3 2 7 0 8 0 8 0}}{3} .
\end{gathered}
$$

And foreseeably,

$$
\cos 2 \Theta_{S T R} \cdot \sqrt{\mathbf{1 2 3 2 7 0 8 0 8 0}}=\exp \exp e \cdot 10^{17}
$$

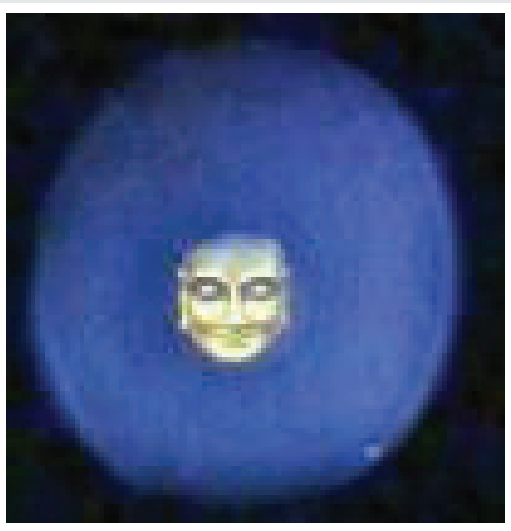

Showing that the entire cosmological evolution reduces to the organic growth process of something absolutely abstract.

The traditional Mongolian metaphysical philosophy declares that apart from cosmic physical forces there is "Great Wisdom and Wit" auspicious to mankind. Now we know what is great wisdom and wit and it is provable that cosmology is, indeed, anthropocentric. 
In the Oriental civilization Buddha Gautama Sakyamuni doctrined that there does exist only Pathway, that is, algorithm, leading to the universal harmony.

Finally, it is a must for us to share the following vital information with the scientific community. Notably that the microelectronic digital technology of ours has by now become compatible with the universal cosmic mathematical machinery. As a result, Cosmos is enabled to hack our digital devices and send to us this life time portrait of Buddha. Consequently, Buddha's immortal soul still browses around in the cosmos to endorse, when time comes, discovery of absolute knowledge by the human intellect under the close guidance by the Cosmos. The Universe as the ultimate known complexity reproduces itself in its micromodel being the human brain. The original and the model can intercommunicate in certain circumstances like the discovery of the superunification theory. Cosmology is a preprogrammed process.

The modern civilization is as fragile as never before. Critical trends in the $21^{\text {st }}$ century threaten to lead to a global catasthrope. To avoid cataclysm and survive on the cosmological scene, the human civilization has at the moment got a good chance. The future of the world will depend upon how promptly the contemporary nations will learn the absolute system of knowledge that unifies both the exact and the spiritual sciences. The only way to derive revolutionary new technologies will be computer simulations of absolute geometry. The world has no more time to be wasted. It is the responsibility of the official science institutions to react to the discovery of the final theory in due manner without fear and prejudice. Time is running out.

\section{References}

1. Libri XY (1558) Euclidis Elementorum. Greace \& Latine. Rome. Link: https://bit.ly/30c1lqk

2. Isaac Newton. General Scholium. In the Principia Mathematica.

3. Whitrow GJ (1961) The Natural Philosophy of Time. London and Edinburgh. Link: https://bit.ly/3HBggl7

4. Smith DE (1929) A Source Book in Mathematics. New-York. Link: https://bit.ly/3oKYLGi

5. Penrose R (2005) The Road to Reality. New-York. A Complete Guide to the Laws of the Universe. Link: https://bit.ly/32guF65

6. Read the unification theory on my website finaltheory.net
Discover a bigger Impact and Visibility of your article publication with

Peertechz Publications
Highlights

* Signatory publisher of ORCID

* Signatory Publisher of DORA (San Francisco Declaration on Research Assessment)

* Articles archived in worlds' renowned service providers such as Portico, CNKI, AGRIS, TDNet, Base (Bielefeld University Library), CrossRef, Scilit, J-Gate etc.

* Journals indexed in ICMJE, SHERPA/ROMEO, Google Scholar etc.

- OAI-PMH (Open Archives Initiative Protocol for Metadata Harvesting)

* Dedicated Editorial Board for every journa

Accurate and rapid peer-review process

* Increased citations of published articles through promotions

* Reduced timeline for article publication

Submit your articles and experience a new surge in publication services

(https://www.peertechz.com/submission). 